\title{
A PROJECTION SCOTOMETER
}

BY

FRANK A. JULER

LONDON

THE mapping out of visual fields by the usual method is handicapped (a) by the sound (b) by the sight of the carrier of the test object. Both of these objections are surmounted by the use of a spot of light projected on to the screen, and various methods have been used from time to time by which this desideratum can be provided.

The principle is used in the Maggiore perimeter made by Zeiss, but the cost of this instrument is too high for most individuals. A similar device, not self-registering, was brought out by John M. Evans for use with a Ferree Rand perimeter, but on the whole the present perimeters in common use are efficient for most clinical purposes.

In examining the central field, however, the method in common use with the Bjerrum screen in this country is to use Traquair's discs of varying sizes. To the discs themselves little exception can be taken save that the smallest $(1 \mathrm{~mm}$.) sizes are apt to be inaccurate in diameter and to be dingy in shade; but the chief objection is the long holder of the discs which, even in its new state, is apt to reflect light from its painted surface, and when worn from use has a definite metallic shine. This may interfere with the accuracy of the record.

From time to time instruments have been made which have been designed for projection scotometry; I found one first at the Central London Ophthalmic Hospital which had been designed by the late Stephen Mayou, possibly six or more years ago; this was manufactured by Messrs. Keeler and formed the basis for the model which is described below, but I can find no record that Mayou published any note of his device.

In 1932 Bishop Harman demonstrated a projection model at the meeting of the British Medical Association. This consisted of two tubes containing filament lamps both of which projected patches of light on to a screen. One of these patches acted as the fixation spot. The other could be varied in size by a series of diaphragms and in colour by filters. Recording was carried out by a prism device, whereby a small beam was diverted from one of the main beams and directed on to a chart. By pencil marks on this chart the field or scotoma was mapped out. I have no personal acquaintance with this apparatus. 


\section{The New Model}

The apparatus now to be described aims at the same result as the Bishop Harman instrument, the test object being a spot of light on a grey surface; the advantage claimed is that it is a less complicated instrument.

It consists of a single tube containing the lamp and arrangement of lenses together with diaphragms that permit of the projection of a circular uniform light area of diameter varying from 1 to $5 \mathrm{~mm}$. on a screen at one metre distance. With these five sizes of test object, a great variation in the stimulus can be obtained, for in addition to the alteration in size the intensity may be varied $(a)$ by altering the resistance of the rheostat and so the illumination of the spot, $(b)$ by altering the general illumination on the screen.

By colour filters red and green images are also provided.

The whole is mounted on a substantial stand, and the lamp tube is free to rotate over an angle of some 60 degrees.

The Screen.-Various screens have been tried. Black is useless for it absorbs the light; white gives rather too bright an illumination; a medium grey is found to be the best. The surface must be matt, or reflexes from the illumination of the room cause error; a smooth grey felt or cloth has given satisfaction. For fixation a circular porcelain white button of $7 \mathrm{~mm}$. diameter is effective.

It is well to have the screen mapped out by silk surface threads giving the tangent angle values at the usual working distance. This is useful (a) as a check on the automatic registration, (b) when the automatic registration is not used. In the latter case the readings are transferred directly by hand to the corresponding map on the field chart.

Automatic Registration.-This is obtained by a pricker on to a chart at the back of the instrument, and is satisfactory.

The instrument can also be supplied in a simpler form without automatic registration, either on a stand or as a simple tube held in the practitioner's hand. The latter has some advantage in that it can also be used as a projector on any simple perimeter which has a grey surfaced arc.

General Illumination.-The examination is best conducted in the dark room with medium illumination on the screen. Care must be taken that the illumination on the screen is uniform and that there is no marked reflection from its surface. It is found that with the grey matt surface two lateral flood lighting lamps with opal glass fronts give satisfactory diffusion.

Method of Use.-The patient is seated comfortably in a chair, his eyes being one metre distant from the screen and slightly to one side of the fixation zero mark. One eye is of course covered. 
The instrument is then adjusted, so that the objective lens at the end of the tube is one metre from the screen, close beside the patient's head. The central registration pin being in the zero position, the projection lamp is switched on and the position of the instrument is adjusted so that the registration zero corresponds with the fixation point. The central pin is then withdrawn and the pricker registration is ready for use.

The image on the screen is focused to a sharp edge and the disc aperture required is turned into position.

In the usual routine I commence by demonstrating the blind spot with a light disc of 2 or $3 \mathrm{~mm}$. diameter, and then search for general depression of the central field or for arcuate scotomata

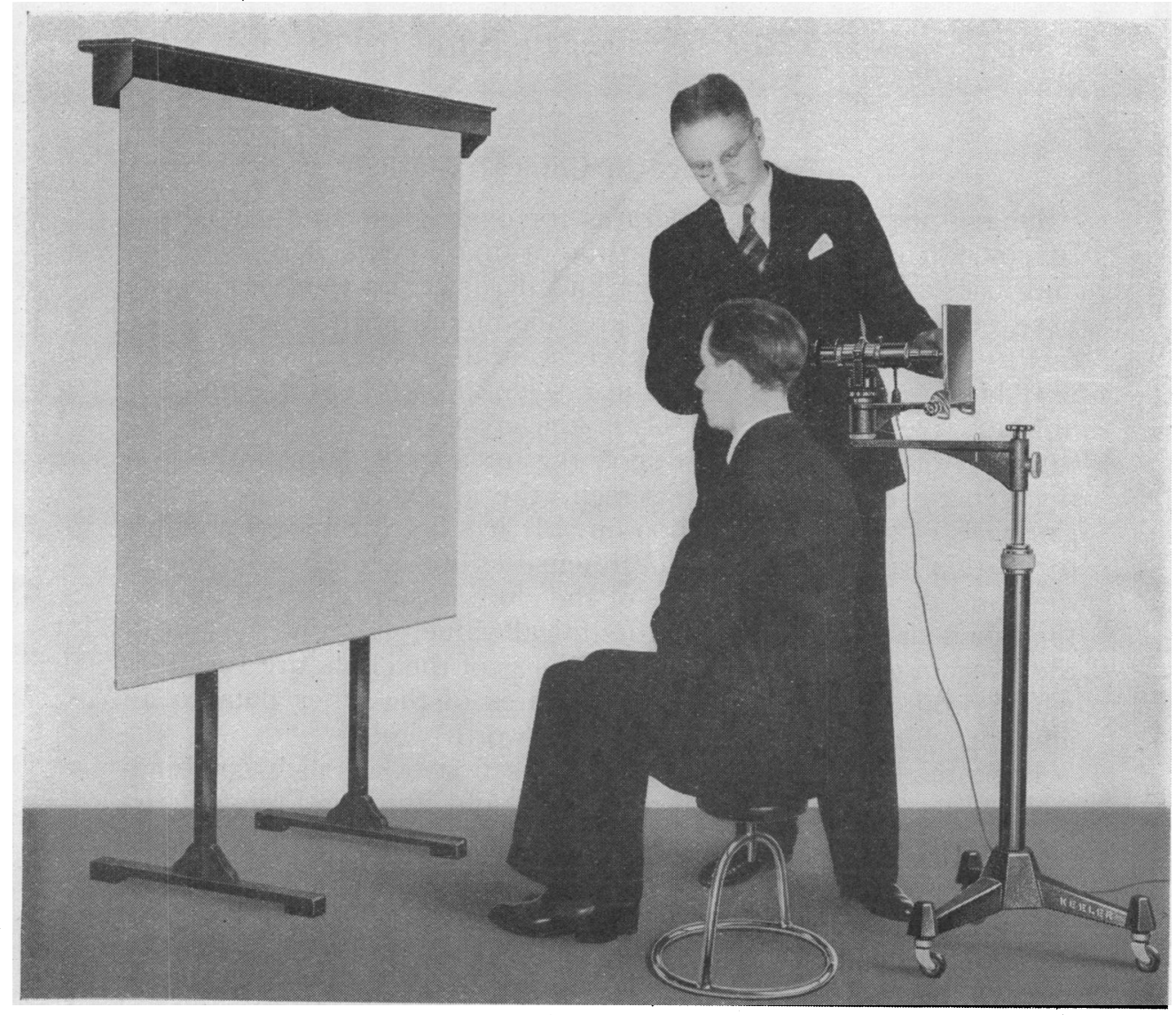

The photograph shows the general appearance and method of use of the projection scotometer (one eye of the subject should have been covered). 
with the same object. The smallest test object can then be used. In tobacco cases the demonstration of caeco-central scotomata for red and green is easy, and, if necessary, the intensity of the colour can be increased greatly by dimming the general illumination falling on the screen.

The criticism may be made that a chin rest is essential for accurate work, but personally I find that this is not necessary, for it is the visual fixation of the zero which is least steady. With the patient in a comfortable chair there is no antero-posterior movement of the head, whilst slight lateral movements do not matter.

The apparatus can of course be used at two metres distance, and has been tested out at this distance by Messrs. Cross and Ayoub, resident medical officers at Moorfields. They report that at that distance the plotting out of the retinal vessels (angioscotometry) is possible; this is good proof of the delicacy of the apparatus.

\section{Experience in Clinical Work}

I have now used this apparatus for over a year and can report that it is a definite advance on the ordinary method with screen and discs on holders. Patients find it easy, and those who have been tested by other methods are unanimous with regard to the facility with which they can give accurate replies.

It has been suggested that for scientific work one should be able to give the variants used in the apparatus, for example the intensity of light in the test spot, the intensity of light falling on the general surface of the screen, the absorption value of the greyness of the screen, and so on. It has been found impossible to do this satisfactorily. The intensities in candle power are so low, and the absorption value of the cloth is so difficult to obtain, that such data would be of no clinical value. Again, the life of the lamp in the tube, and the cleanliness of the cloth, are variables which would vitiate the delicate figures of the other data to an amount which would not make them worth while.

For practical purposes, one requires first of all such combination of size and intensity of test object and illumination of screen which to the normal eye gives a visual field of $10^{\circ}$ to $15^{\circ}$ only. That is easily arranged when the apparatus is in place by adjusting the rheostat and the general screen illumination whilst testing several normal individuals with the smallest aperture. This then is the minimal stimulus required, and the larger apertures will suffice for the stronger stimuli.

Mr. Charles Keeler is responsible for the design and manufacture of the instrument. 